\title{
Théologiques
}

\section{Professer et confesser}

\section{Penser des espaces théologiques à partir de Jacques Derrida et Thomas d'Aquin}

\section{Maxime Allard}

Volume 14, numéro 1-2, automne 2006

Les lieux de la théologie aujourd'hui

URI : https://id.erudit.org/iderudit/014316ar

DOI : https://doi.org/10.7202/014316ar

Aller au sommaire du numéro

\section{Éditeur(s)}

Faculté de théologie et de sciences des religions, Université de Montréal

ISSN

1188-7109 (imprimé)

1492-1413 (numérique)

Découvrir la revue

Citer cet article

Allard, M. (2006). Professer et confesser : penser des espaces théologiques à partir de Jacques Derrida et Thomas d'Aquin. Théologiques, 14(1-2), 143-160. https://doi.org/10.7202/014316ar

\section{Résumé de l'article}

Afin de construire un protocole heuristique pour questionner les manières de parler des «lieux de la théologie» en rapport avec l'université, cet article propose un parcours inspiré de Derrida. Ayant installé le questionnement épistémologique autour des verbes "professer» et «confesser», il fait remarquer la complexité des rapports entre ces fonctions. Il inscrit ensuite ces rapports sur le double horizon historique des réflexions allemandes sur l'université à l'orée du XIX ${ }^{\mathrm{e}}$ s. et de l'université médiévale et ses marges. Ce double horizon déplace le questionnement vers les relations entre les professeurs et les étudiants, relations portées par le désir et la promesse de raison comme constitutifs de lieux théologiques dépassant les cadres institués de la faculté de théologie pour se retrouver en jeu dans toute l'université.
Tous droits réservés @ $\odot$ Faculté de théologie et de sciences des religions, Université de Montréal, 2006
Ce document est protégé par la loi sur le droit d'auteur. L'utilisation des services d’Érudit (y compris la reproduction) est assujettie à sa politique d'utilisation que vous pouvez consulter en ligne.

https://apropos.erudit.org/fr/usagers/politique-dutilisation/ 


\title{
Professer et confesser \\ Penser des espaces théologiques à partir de Jacques Derrida et Thomas d'Aquin
}

\author{
Maxime Allard, o.p. \\ Collège dominicain de philosophie et de théologie
}

En 2001, dans L'université sans condition, Jacques Derrida tissait son propos sur les pratiques universitaires sur la trame de vocables d'origine « religieuse » : professer et confesser (2001). Il reprenait ainsi, et à nouveaux frais, ses élaborations sur l'institutionnalisation du savoir dans $D u$ droit à la philosophie (1990). Partant de ces analyses, il s'agira de réfléchir sur la constitution du caractère "public », institué, du lieu des dires théologiques à moins qu'on ne préfère lire «des lieux de la profession théologique » ou encore «des lieux des confessions théologiques». Serviront de balises pour cette réflexion les transformations des postures "professantes » et "confessantes" dans les divers types d'universités (médiévaux, allemands des Lumières et du romantisme), à la fois dans les facultés (théologie et autres) et dans leurs rapports à d'autres institutions sociopolitiques et ecclésiales. Au nom donc d'un quasi-idéal critique, il y va d'un éclairage renouvelé sur le phénomène universitaire, concentré sur les «Humanités ", les humanities, les sciences humaines... voire quelque chose comme ce qui s'est appelé, depuis l'institution médiévale universitaire, de la théologie et ses mutations, reconfigurations ou reconversions possibles en sciences religieuses, sciences des religions.

Pour radicaliser mon propos, je ferai les deux propositions suivantes; à moins qu'il ne soit plus juste - mais alors cela ne sera justifiable qu'en fin de parcours - de parler d'une seule proposition en deux volets qui marquerait alors une tension interne au thème de ce numéro: le(s) lieu(x) de la théologie. Voici, en une formulation proche de l'exclusion: la théologie n'a son lieu (propre) à l'université ni au Moyen Âge ni depuis; l'université est déjà toujours un lieu structuré par (de) la théologie, des options théologiques. Dans ce texte-ci, il ne sera pas possible de conclure. Je ne 
ferai que tenter de décliner des questions... À la suite de l'élaboration de cette double proposition instable, je signalerai un modèle autre d'élaboration de l'espace/temps théologique en m'inspirant tant de Derrida que de Thomas d'Aquin.

Pourquoi une telle approche? Dans quel but? Déverrouiller, débloquer le questionnement, déjouer des fantasmes. D'une part, elle permet de ne pas enfermer la discussion et les intervenants dans les seuls cadres actuels des questionnements sur l'avenir des facultés de théologies, sur leurs reconfigurations (possibles et/ou nécessaires) en lien avec des pratiques en provenance de sciences dites des «religions » ou « religieuses ", discussions où il en irait du caractère "naturel » de la théologie universitaire. Cette approche permet, du même coup, de déjouer la mythologie accompagnant la discussion soit en provenance d'un fantasme romantique catholique «médiéval » où la théologie serait fondatrice et/ou à l'origine du mode de réflexion universitaire, soit en provenance de discours sur les rapports supposés universels et contraignants entre de la «foi » et de la « raison» (de Libera 2003). D'autre part, cette manière de déborder les cadres habituels permet de signaler quatre dimensions du problème des lieux de la théologie souvent occultées :

a) la possibilité de lieux extra-universitaires pour la production de discours/pratiques théologiques, voire déjà l'opportunité de reconnaître l'existence de ces lieux, ecclésiaux ou non, et de penser les rapports complexes entre leurs productions et les productions universitaires autrement que comme un rapport de pouvoir et de censure exercé par la faculté universitaire au nom d'une certaine rationalité et d'un certain protocole corporatif;

b) un rapport à repenser et, probablement, à instituer à nouveaux frais, entre le lieu des pratiques du croire ecclésial et ceux de la reprise réfléchie de ces pratiques et de leur insertion universitaire;

c) le caractère corporatif professé du travail théologique et les implications sur ce travail des modes de constitution de cette corporation sur la "profession », la professionnalisation et la «confession », mais aussi sur ce qui a lieu dans l'enseignement, dans le rapport aux personnes inscrites «en théologie» (Bourdieu 1989a ; 1989b; Bourdieu et Passeron 1970);

d) la question de l'éthique de l'engagement et du désir impliqués sur les lieux théologiques, à moins, comme on le suggérera en fin de parcours, que l'éthique du désir engagé ne soit à l'origine de la localisation du partage théologique. 


\section{Du statut de la double proposition}

\subsection{La théologie n'a pas son lieu (propre) à l'université.}

Cette phrase sous-entend presque déjà ceci: le positionnement, l'énoncé positif primitif d'une thèse (la théologie a son lieu propre à l'université) en même temps que la reprise critique ou, mieux, la prise qui nie cette thèse. Cela pourrait avoir lieu selon divers protocoles:

a) mettre en cause la notion même de lieu propre, de lieu «naturel » au nom du caractère problématique du "propre» ou au nom de la disqualification moderne (en physique à l'origine) de toute cette problématique physique aristotélicienne liée à une certaine construction du monde comme cosmos depuis les Grecs;

b) soumettre à la question l'idée même selon laquelle il y aurait ou devrait y avoir "un » lieu de la théologie ou que la théologie serait une de par ce lieu (l'université) ou de par une nécessité liée à son statut épistémologique;

c) dans un style proche du Lyotard du Différend (1984), insister pour marquer le statut de cette phrase (cognitive, performative, "symbolique ", sublime) afin de déterminer le protocole approprié pour l'analyser et la proposer en tenant compte - injection derridienne — de l'instabilité interne qui la grève déjà, de sorte qu'il faille trancher sans détermination assurée ;

d) mettre en cause le rapport de la théologie à l'université. Compte tenu de l'espace imparti ici, je n'insisterai désormais que sur le dernier protocole.

\subsection{L'«université» est déjà toujours un lieu structuré par (de) la théologie.}

Quelques remarques rapides soulignent le caractère plus complexe de cette seconde phrase, qui complète, inquiète et nargue la première. Il y a le "déjà toujours ", premièrement, qui rend impossible l'exclusion de la théologie et de l'université. Puis, il y va de l' "université », qui se retrouve entre guillemets, car il n'y aurait peut-être pas une université, mais une idée de celle-ci, dans l'attente de l'université à venir, pour emprunter encore à Derrida parlant de "démocratie à venir " (2003, 153s.), à moins qu'il ne s'agisse simplement de noter que le mot "université " peut recouvrir et renvoyer à des expériences historiques diverses, irréductibles les unes aux autres. Plus radicalement, autour de cette " université », entre guillemets, se pose la question de la possibilité d'un lieu, d'une telle entreprise aujourd'hui, désormais: les 
savoirs s'organisent-il en un univers ? Peuvent-ils s'articuler ou être articulés pour produire une unité quelconque? Et en fonction de quels protocoles? Et par qui ? L' " université » ne serait-elle pas un rêve idéaliste dont il faudrait se défaire ou qu'il faudrait convertir? Serait-elle un événement à attendre, sans pouvoir adéquatement le préparer, s'y préparer ? Serait-elle à annoncer ? Enfin, il y a le «de » qui se retrouve entre parenthèses pour permettre une double lecture de la finale de la phrase, double lecture qui devrait et ne peut se faire en même temps. Qu'entraînent ces remarques? Qu'il n’y a pas «la théologie ", mais des pratiques théologiques ou du "théologique » indépendamment de la présence ou non d'une faculté ou d'un département de «théologie» (et/ou de sciences religieuses); qu'il demeure, pour qu'il soit possible de reconnaître une "université ", quelque chose comme ce genre d'institution, des principes théologiques. Dans ces conditions, au cœur ou dans les marges de l'université, le rôle spécifique de pratiques théologiques serait de rappeler la fragilité épistémologique disséminée sur le campus et l'impossible fondation - au sens derridien $(2003,123)$ — pourtant désirée par le discours, sa corporatisation et sa professionalisation.

\section{Des places modernes pour des pratiques théologiques}

Depuis les Lumières franco-allemandes, mais cela fut surtout thématisé par l'Allemagne, la place de la théologie à l'université et, particulièrement, son ton, est devenue problématique ou, mieux, aura été problématisée.

Il importe cependant de noter un déplacement préalable trop souvent occulté. D'autant plus oblitéré qu'il eut lieu aux limites de la philosophie et de la théologie: Descartes avait habilement fait l'épochè des pratiques théologiques sorbonnales... tout en s'adressant à leur autorité ${ }^{1}$ ! Malebranche, de l'Oratoire, entretint des liens complexes avec la Sorbonne. Qu'il suffise de mentionner ses querelles avec Arnauld! Pour leur part, Leibniz et Spinoza, sur le continent, Hume, Locke et Berkeley, dans les îles britanniques, avaient aussi déplacé les lieux où des discours à teneur théologique pouvaient circuler et être produits. Il faudrait aussi certainement retracer les liens, les procédés auto-immunitaires et les «contaminations» entre, d'une part, les universités scolasticisantes et étatiques et, d'autre part, les lieux de productions historiques tels ceux des Mauristes (avec l'impact de la méthode pratiquée par Mabillon) et ceux des Bollandistes.

1. Il serait aussi intéressant d'étudier, en milieu protestant hollandais, les troubles universitaires et civils dont Descartes fut l'objet et dont il fit les frais entre 1637 et 1649. 
Mais concentrons notre attention sur les transformations des pratiques théologiques depuis les Lumières allemandes. En effet, on peut déjà en lire les options dans la première préface de Kant à son ouvrage La religion dans les limites de la simple raison, de la raison dénudée, révélée, dépouillée de tout oracle ou pressentiment (Ahnung) néoplatonicien et de ses guises "chrétiennes» (Fenves 1998). Il y allait de partage des tâches entre le théologien biblique et le philosophe, à l'ombre de la puissance tutélaire étatique. À l'époque, Kant avait déjà établi sa distinction heuristique entre le public et le privé: entre, d'une part, la possibilité de publier une réflexion critique dans l'espace public virtuel constitué et institué par le régime de lecture et de circulation mis en place par les périodiques savants et, d'autre part, le travail de fonctionnaire de l'individu ayant pour fonction de faire circuler ce que l'Institution théologique requérait de lui. Cela remontait à sa réponse à la question: Qu'est-ce que les Lumières? Et, déjà, à cette occasion, Kant notait les limites anthropologiques de l'écartèlement entre le public et le privé lorsque ce clivage traverse la même personne. Mais il faudra attendre Le conflit des Facultés pour que Kant traite et négocie ouvertement avec les facultés de théologie, de droit et de médecine, du statut de leurs discours, des effets de ces discours dans l'État. Il concentra son traitement sur la place et le rôle de la théologie. Pour donner des limites critiques à l'extension, au ton et au propos théologique. À la philosophie la faculté qui comptait l'histoire, la philologie, les mathématiques et, aussi, la philosophie - était assigné le rôle critique, le rôle proprement public. Les théologiens avaient un rôle de fonctionnaires d'un État qui avait besoin de personnes pour traiter un corpus précis, autorisé par l'État et s'autorisant, de manière transcendantale, grâce à une foi en la révélation. Le but: instituer des personnes capables de répondre à la question anthropologique, existentielle, question qui témoignait d'une certaine paresse et d'un certain mal égoïste des humains portés par leurs inclinations naturelles. Tâche sociale et politique de la théologie, fonction éthique parergonale dans l'espace de l'agir humain. Des théologiens, individuellement pris, pouvaient toujours participer à l'espace virtuel philosophique de discussion, mais ils se clivaient alors et risquaient toujours la censure, la perte d'emploi. Mais, pour la faculté de théologie, en tant que telle, comptait seulement sa fonction pratique, pédagogique, sociale.

Par la suite, la mise en place et les réformes de l'université allemande en lien avec l'idéalisme allemand, la création de l'université de Berlin, ne devaient pas se satisfaire de l'option kantienne. Tour à tour, Schelling, 
Fichte, Hegel, Schleiermacher et Humboldt, à divers titres, vont écrire à ce sujet. À chaque fois sont en jeu la place de la faculté de théologie, son ton, sa fonction dans le registre du "savoir» et du concept et dans celui de la pratique $^{2}$. Il y a certes une option plus "libérale » orchestrée par Schelling, Schleiermacher, Humboldt, et une autre plus «autoritaire» (selon l'expression de Ferry-Renaut) en la personne de Fichte. Mais, à chaque fois, il y va d'une insatisfaction certaine avec la pratique théologique. Ainsi par exemple, Schelling déplorait sa transformation en philologie et en histoire, transformation portée par le fantasme de l'origine, comme s'il en allait là de la vérité de l'enjeu théologique. Pour lui, la théologie, très restructurée par la philosophie, aurait dû donner lieu à une approche proprement spéculative en lien avec l'idée même du christianisme comme vecteur civilisateur et révélateur de la vérité. Fichte, pour sa part, demandait une conversion complète de la théologie vers sa véritable essence, une sortie hors de sa logique du secret, une séparation consentie de sa partie pratique (éducation du peuple) et de sa partie scientifique pour qu'elle puisse prendre place dans ce qu'il nomme « une école de l'usage de l'entendement » ou du «savoir» (Collège de Philosophie 1979, 193, 198). Il s'agissait donc de localiser à l'université ce qui pouvait s'y localiser comme savoir historique, philologique, et de le disséminer dans la faculté de philosophie au sens kantien. Et, éventuellement, au moins selon Kant et l'État de son époque, de garder la faculté de théologie comme lieu de transmission pratique d'injonctions pour faire vivre.

On sait l'impact de cette modernité allemande sur les transformations des pratiques théologiques et de ses lieux du côté catholique. Cela a bien été étudié: transformation des écoles romaines de théologie et de leur statut pour le reste de l'Église catholique (McCool 1989; 1992). Sans compter les

2. Les textes sont réunis dans Philosophie de l'université. L'idéalisme allemand et la question de l'université, textes de Schelling, Fichte, Schleiermacher, Humboldt, Hegel (Collège de Philosophie 1979). Pour Schelling, il s'agit surtout des huitième et neuvième leçons (107-122) des Leçons sur la méthode des études académiques; pour Fichte, des $\mathbb{2} 2$, 26-27 (193-201) de son Plan déductif d'un établissement d'enseignement supérieur à fonder à Berlin, qui serait en liaison intime avec une académie des sciences; Schleiermacher, dans ses Pensées de circonstances sur les universités de conception allemande, chap. 4 sur les facultés; Humboldt, pour sa part, dans son Sur l'organisation interne et externe des établissements scientifiques supérieurs à Berlin, traite la théologie rien; Hegel, outre des passages dans ses Leçons sur la philosophie de la religion et dans la Phénoménologie de l'esprit, mais aussi De l'enseignement de la philosophie au lycée, dès le cours élémentaire il y va de l'étude de la religion comme couronnant l'étude du droit et de la morale (333-334). 
vives tensions institutionnelles au cours de la crise moderniste. Ce qui prépara les transformations récentes et actuelles de la fonction et de la place de la théologie dans les universités ici - dont les débats autour des travaux de McCutcheon et Strenski sur la manufacture de la «religion» en sont un indice (McCutcheon 1995; 1997; 2001; voir Idinopulos et Wilson 1998; Idinopulos et Yonna 1994).

Pour comprendre l'enjeu de ces diverses options et prises de positions quant à la présence de la théologie dans l'université moderne, la question de la «profession » et de son rapport à la «confession" s'avère utile. Il y va de la possibilité reconnue et reconfigurée diversement pour la «confession " d'être professée sur le lieu universitaire. La "confession" devient ainsi un objet pour les tractations conceptuelles de l'université grâce à une transformation de la posture même de la "profession ". Ainsi, le passage de la profession comme redoublement de la confession à sa redéfinition comme travail négocié selon un code n'est plus enraciné dans le geste confessant lui-même. Il y va toujours d'engagement impliqué dans l'acte de confesser et de la responsabilité qui s'y love. Mais, désormais, cet engagement n'est plus premièrement ecclésial, le gage est offert à une instance de savoir, elle-même portée et initialisée par un geste politique. Il importe de noter le transfert du geste de profession de l'Église et de communautés confessantes à une institution qui, bien que se soutenant d'un discours de la raison et des idéaux de la raison, et bien que s'adossant au pouvoir politique pour s'y soutenir, va demander une loyauté professée envers ces idéaux et les discours les rendant possibles. Cette profession et les diverses professionnalisations qu'elle entraîne demeurent enracinées dans une certaine confession, dans une confiance, dans une foi engagée: engagement dans des pratiques de production de savoir, confiance dans les capacités de la raison ou de l'entendement humain à s'engager dans de telles pratiques; foi dans l'effet de ces savoirs produits, dans l'effet bénéfique de leur circulation, de leur mise en circulation gérée politiquement. Autant d'éléments lentement négociés par Derrida dans L'Université sans condition. Nécessaires conflits de confessions? Peut-être pas, puisque la matrice structurelle de «fiance » est la même: d'un côté comme de l'autre, il y va d'un Idéal délocalisé et universalisant (Dieu, la Raison); d'une localisation particulière de l'expression et de l'articulation de cet Idéal universalisant sur un territoire plus vaste, lui-même déjà marqué par des traces de cet Idéal, traces à faire remarquer grâce à ce lieu particulier (Églises, universités); d'autorités historiquement localisées et de modes de transmission autorisés. Surtout, à chaque fois, il y va d'un clivage, d'une tension entre deux types de confessions 
en tant qu'expressions articulées: la foi du charbonnier et la foi réfléchie/ réflexive (Église, pratiques théologiques), opinions et concepts (société, discours universitaires). Enfin, peut-être trop souvent oublié, mais rappelé par Derrida, il importe de signaler l'horizon «mystique " à l'Idéal délocalisé: il y va de la tension, sur l'espace d'expérience autorisé par cet horizon, entre la notion de travail/métier et celle de "profession» où résonnerait déjà toujours celle de (con-) "vocation », elle-même pressentie par l'idée d'expérience, de choc convertisseur. Ce premier élément «mystique " possède maintes conséquences quant à la temporalité du geste universitaire et, du coup, sur la présence et la tonalité de l'adresse aux étudiant(e)s. De plus, sur cet horizon, il y va aussi de la «confession » joyeuse ou réticente des limites de validité du discours argumenté en cours de profession: entre l'Idéal et ses revêtements et véhicules particuliers, historiquement déterminés, il y a toujours écart, écartèlement, différance à signaler, à confesser. À l'horizon, pour ne pas dire au cœur du geste même, il en va donc d'une certaine démaîtrise, de la reconnaissance d'une certaine passivité. Difficile confession des limites...

Dans cette situation, la présence de la théologie est de type socratique. Comme un taon, elle pique, célèbre ouvertement ce que l'université pourrait vouloir oublier, faire oublier ou ne voudrait surtout pas entendre énoncer dans des termes signalant la fragilité de son propre discours. Ainsi, le déplacement de la théologie vers des sciences religieuses, ou sciences de la religion, n'y changerait rien ou changerait tout. N'agaçant plus, elle pourrait durer sur un lieu, professant désormais ne pas professer, professant selon des normes de droit (enseignement, recherche, travail) au moins partiellement déconnectées du geste même que ce droit tente de protéger.

Permettez un dernier tour d'écrou concernant le discours théologique et sa présence incontournable à l'université, malgré la marginalisation plus ou moins effective des facultés de théologie. Dans ce cas, il y va déjà toujours de la théologie dans les divers lieux où ça parle en Occident, où ça cherche à savoir, dans le cadre professionnel théologique ou non. Il en irait de faire redécouvrir alors aux parlants d'Occident la profession de foi impliquée, la fiance - déclarerait Derrida (Derrida et Vattimo 1996, 7485 ) - dans la parole, dans son partage et celui des tons et des voix qu'elle permet, de ce qu'elle partage et de ce à quoi elle donne part ${ }^{3}$. Dans ce

3. Sur cette question, il faudrait longuement commenter le travail de Jean-Luc Nancy sur la déconstruction du christianisme et les réticences derridiennes sur la possibilité d'achever ce geste. 
sens, de toutes parts, l'université serait enserrée par de la production théologique! Malgré elle...

\section{Des places médiévales des discours théologiques}

Comme, dans ce contexte, on argumente parfois du caractère originaire de la théologie à l'université et qu'on en conclut à sa nécessaire présence institutionnelle sur le campus universitaire, il convient en second lieu d'en explorer rapidement l'histoire (Le Goff 1999; 1977). Cela permettra d'engager autrement la question du lieu. Pas question ici de refaire l'histoire qui va de Salerno à Paris en passant par Bologne (Haskins 1990). Simplement de souligner que le lieu propre de l'université alors est l'association des maîtres et des disciples (societas magistrorum discipulorumque). Il aura été constitué, d'une part, pour que maîtres et disciples puissent se protéger économiquement de la ville dans laquelle leur communauté se réunit. Mais à ce facteur immunitaire il faut joindre celui des relations internes à la communauté, au collegium, où il y va aussi de définir, déterminer et protéger les droits et devoirs des professeurs - avec l'élément pécuniaire de leur engagement, "negotia scholaria» (Derrida 2001, 64) — et des étudiants, de signaler la constitution de "nations ", comme quoi il y aura eu, très tôt, quelque chose de "cosmopolitique " dans l'univers universitaire et non simplement de «national » selon le modèle franco-allemand moderne ${ }^{4}$. Au nom d'une certaine profession confessée publiquement, il en va d'une séparation, de la prise en compte de ses aspects économiques (registre monétaire et celui des échanges de savoirs), du prix de la vérité, de la constitution de la confiance en ces structures. Au moins pour le cas de Paris, (trop ?) souvent pris comme paradigmatique, on assiste à une lente mutation de l'école cathédrale et des écoles environnantes vers ce qui devient l'université, par un complexe réseau de sociétés, de réglementations internes marquées, confirmées ou ordonnées tant par des privilèges et orientations royaux que par d'autres pontificaux au tournant du XIII ${ }^{\mathrm{e}}$ siècle (Verger 1997; Rashdall 1936). Déjà là, mais autrement que dans la modernité éclairée du XIX ${ }^{\mathrm{e}}$ siècle, le pouvoir politique se signale pour se démarquer du pouvoir ecclésiastique ou pour le doubler.

4. Il faudrait analyser aussi comment les villes, «églises » et pouvoirs politiques se sont protégés de l'institution universitaire et comment ces processus et procédés d'immunisation ont varié selon que l'on s'adressait à telle ou telle faculté. 
Mais cette université n'est pas le seul lieu de la production théologique. Il faut compter avec une certaine émulation entre l'université naissante et l'organisation de centres d'études (studium) dans les ordres mendiants qui se répandent, surtout l'Ordre des Prêcheurs et les Franciscains - les aspects économiques n'étant pas absents de ce rapport critique et devenant vite conflictuels sur fond de la nature même et la finalité de la théologie en tant que sacra doctrina (Mulcharey 1996). Un rapide tour d'horizon fait prendre conscience que c'est dans les marges de l'institution universitaire ou dans les chaires au statut ambigu que les négociations théologiques marquantes ont pris naissance, se sont structurées avant d'être ré-appropriées, tardivement parfois et avec une certaine dénaturation, par les universités ellesmêmes. Ainsi, à l'origine, si du moins on pense à un monument comme la Summa theologice de Thomas d'Aquin, ces gestes théologiques n'y étaient pas destinés comme à leur lieu propre. En effet, le père Boyle a bien montré, jadis, que la Summa theologice fut produite à la demande des autorités dominicaines pour les étudiants dominicains qui n'iraient pas à l'université et que le gros de cet ouvrage - en tous les cas du point de vue des manuscrits copiés et conservés et de l'usage prévu par les actes des chapitres ayant incité à son écriture - porte sur l'éthique, une éthique de la confession et de l'accompagnement (Boyle 1982, 30) ! Le tout, dès l'orée du texte, porté explicitement par l'acte d'élaguer les méandres des tractations et arguties universitaires.

Ainsi, autour de la professio et de l'activité de professare, dans la querelle entre les séculiers et les mendiants, un curieux conflit de professions a lieu : selon les séculiers, les religieux, définis par une profession, désignés comme "profès » (c'est-à-dire ceux qui font une profession solennelle) ne peuvent être professor ${ }^{5}$. On ne pourrait professer à l'université si on a professé, si on fait profession d'une règle religieuse (professio regulce). Seraitce parce que, à l'inverse, l'université aurait une profession déréglée ? Certes non. Sa règle serait-elle dérèglementante pour les règles externes, première et exclusive par rapport à elles ? Partiellement au moins. Il y aurait donc déjà confession d'une autre règle, celle d'une certaine raison, elle-même conçue, de manières diverses - franciscains et dominicains divergeant sur ce point entre eux et avec les séculiers. Cette raison serait marquée et portée par (de) la transcendance "divine", son excès et sa sagesse. Dans ces conditions, la rationalité confessée et professée ne serait pas étrangère à ce

5. Summa theologice, IIaIIæ, q. 188, a. 5, obj. 3. 
qui se donnait à penser de par Dieu, grâce à Dieu, dans l'aire ecclésiale. Ce paradoxe se signale sur un territoire donné, doublement institué par le sceptre et la crosse, par des privilèges royaux et d'autres épiscopaux et pontificaux.

Force serait donc de conclure ce parcours sur le lieu universitaire d'inscription de la théologie de la manière suivante: la pratique de la théologie, en tant qu'elle serait portée explicitement par une profession religieuse, aura toujours été dans un rapport instable et problématique avec la matrice théologico-politique qui structure le lieu universitaire. Et ce, que l'analyse porte sur le moment médiéval de cette situation alors qu'était partagée, par tous les acteurs, la confession chrétienne, ou qu'elle porte sur l'époque moderne où le partage de la "confession " a été complexifié et joue sur des registres différenciés, opposés. On pourrait être tenté de situer le problème sur le plan épistémologique et d'y voir, dans ce champ, la mise en place de rapports complexes entre l'universel confessé, désiré, espéré, et le particulier professant, tant comme individu et/ou lieu institué concret que comme énoncé déterminé. Il en irait alors du paradoxe d'une nécessaire inscription de la confession de l'universel par un individu situé dans un lieu institué lui offrant déjà toujours un protocole universalisant de ses énoncés. Resterait alors à négocier l'exposition de ce paradoxe et de ses conséquences. À chaque fois, le mode théologique de cette exposition, dans son caractère hyperbolique, dans son recours à de la «transcendance» risque de déstabiliser les économies particulières déjà en place.

Prenant acte de cette situation, il est désormais possible de tenter une approche différente de la constitution de lieux de productions théologiques. Je le ferai à partir d'un croisement de l'espace et du temps, moins en fonction de lieux déjà constitués, reconnus, institutionnalisés qu'à partir des acteurs même (se) partageant du savoir en production. Du coup, il s'agira d'arrimer le registre épistémologique à celui d'une éthique de l'énonciation marquée par du désir. Et pour le faire, la structure complexe de l'entrée dans la Summa theologice s'avère opportune.

\section{De la constitution d'espaces/temps de la pratique théologique}

Parce que le docteur de la vérité catholique ne doit pas seulement instruire les plus avancés, mais qu'il lui appartient aussi de sortir de l'état brut les commençants, selon ces mots de l'Apôtre $(1$ Co 3,1$)$ : «Comme à de petits enfants dans le Christ, du lait je vous ai donné à boire, non de la nourriture solide ", le propos de notre intention est dans cet ouvrage, de faire tradition 
de ce qui concerne la religion chrétienne de la façon la plus convenable à l'érudition des commençants. Nous avons considéré en effet qu'il se trouve dans l'emploi des écrits des divers auteurs plusieurs éléments qui empêchent les novices de cette doctrine: en partie par la multiplication des questions inutiles, des articles et des arguments; en partie aussi parce que ce qui leur est nécessaire en vue de la science n'est pas traité selon l'ordre de la discipline, mais selon que requiert l'explication des livres, ou selon que se présente l'occasion des disputes; en partie parce que la répétition fréquente des mêmes choses génère dans l'âme des auditeurs lassitude et confusion. Pour éviter donc ces inconvénients et d'autres semblables aux étudiants, nous tenterons, avec confiance dans le secours divin, de suivre ce qui a trait à la doctrine sacrée brièvement et clairement, autant que le permettra la matière.

Ce prologue à la Summa theologice définit le lieu de la pratique théologique moins par un espace physique à occuper, un lieu ou une institution, sa corporation et les rapports de forces qui s'y engagent, son horaire et ses règlements, que par un espace confessé professé - pour éviter de le décrire comme un "espace symbolique — - celui de la religion chrétienne et de la vérité catholique. Là s'organisent des échanges de parole entre des instances occupant diverses positions et postures, en tant que ces échanges sont médiatisés par des écrits, des questions énoncées, des demandes adressées. La profession de foi chrétienne y joue un rôle constitutif.

Pourtant, d'une part, cet espace lui-même est structuré par le désir disséminé sur divers pôles, désir qui fait se croiser ces pôles, via la parole et l'écrit. D'une part, il y a le désir d'apprendre de qui commence, du "novice ». Ce désir ne doit pas être éteint, lassé, dégoûté, mais il est à "satisfaire » en en faisant assez et de telle manière que le nouvel arrivant sur la scène de ce désir, sur cette scène du désir de vérité catholique, désire continuer à être érudit (erudire), soit littéralement, à se (faire) dégrossir, se (laisser) raffiner, affiner sa pensée, à y durer et y croître pour s'apprêter à ne plus recevoir du petit lait épistémologique et cognitif! De l'autre côté, il y a aussi le désir du "docte » engagé dans un travail d'exposition où il y va toujours d'exposer la vérité en s'y exposant. Ce désir est complexe car, d'emblée, il porte une dynamique propre de la surenchère, de la surexposition, de l'opposition à d'autres expositions, à d'autres professants. Autant de risques de lasser et de saturer le désir du nouvel arrivant sur la scène. Désir qui, du coup, risque d'empêcher la profession, l'engagement au partage responsable de la vérité et de sa jouissance.

D'autre part, et à cause de cette structure de partage désiré et désirant, il en va d'une certaine complexité du statut épistémologique même de l'acte 
théologique et de l'acteur théologique professionnel, donc de la possibilité de son institutionnalisation dans un lieu défini comme lieu de production et de transmissions de savoirs. En effet, l'acte théologique n'est pas premièrement marqué par ce qui relèverait du "constatatif ", mais du "performatif ". Malgré l'ébranlement derridien de cette distinction (Derrida 2001, 73 ; 2003, 209-210), il y a performance théologique. Premièrement quant à qui professe faire acte de «docteur » et ce, antérieurement — logiquement - même à la constitution du lieu institutionnalisé lui-même ${ }^{6}$ : il y a promesse de faire, d'affermir et de renforcer ce vouloir-faire en-vers autrui, ce propos même de professer et d'en répondre publiquement, devant témoins, alors que cette publicité, en soi n'est pas nécessaire. La promesse a aussi ici pour fonction d'exciter le professant ${ }^{7}$ et, par ricochet, d'exciter qui vient adresser une demande, exposer son désir de connaître à ce professant et, ainsi, de le maintenir dans ce désir de s'adresser pour que de la vérité lui soit adressée en retour. La profession est prise de parole une fois pour toute devant autrui pour signaler que désormais tout l'agir aura été (en)gagé ad Deum, en-vers Dieu, ce qui entraîne un mode de vie, un rythme de travail professionnel où le don est premier, où il s'agit de s'y adonner en s'y donnant. La profession est donc le complexe acte délibéré d'un projet promis: là où le volo, désir raisonnable délibérément réfléchi, devient votum qui, avant d'être public, est déjà comme un témoignage de soi envers soi, occasion d'une accession désirée en posture subjective. Ce geste concomitant à celui du novice qui désire s'adresser entraîne ce novice dans la profession. En ce sens, la profession théologique devient alors l'acte commun du professeur et de l'étudiant, le temps de leur désir, le temps requis pour le satisfaire.

Deuxièmement, il y va aussi de la théologie et de son lieu comme d'une performance débordant toujours déjà la production/transmission de savoir selon un horaire et des règles négociés. On en trouve la trace dans l'instabilité constitutive du statut épistémologique de la théologie lorsqu'elle contribue à instaurer et inaugurer les régimes universitaires. Qu'il suffise de rappeler, puisqu'il n'est pas temps ici de l'analyser, la logique de la question première de la Summa theologice: l'unité de cette pratique fait problème dès l'abord de sa scientificité ${ }^{8}$; une fois son unicité transcendentale et heuristique professée

6. En effet, dans la Summa theologice, la question de la profession et du votum la structurant (IIaIIæ, q. 88) est négociée antérieurement à la constitution du traitement des «états de vie» (IIaIIæ, q. 188). Cet écart structurel est symptomatique.

7. Thomas d'Aquin, Summa theologice, IIaIIæ, q. 88, a. 1.

8. Summa theologice, Ia, q. 1, a. 2-3. 
et arguée, il s'agit de savoir si le discours théologique relève de l'ordre du théorétique ou du pratique. Options thomasiennes contre la tradition assumée par les écoles franciscaines naissantes, qui remarquaient son caractère pratique au point de déterminer l'énoncé de la mise en question: Videtur quod sacra doctrina sit scientia practica. Réponse : elle est une science théorique et pratique: sacra tamen docrtina comprehendit sub se utrumque, avec une valence plus théorique où il y va de préserver la raison, d'être responsable raisonnablement et de manière délibérément professée. L'activité humaine est elle-même envisagée comme médium pour connaître Dieu, ce qui est l'accomplissement jouissif de cette activité humaine même ${ }^{9}$. Donc discourir théoriquement est, de soi, une activité qui permet la béatification, la jouissance.

Cependant, ceci à peine soutenu, dès lors que la théologie est mise en rapport avec les autres discours, se pose la question de son caractère critique et fondateur, dirions-nous, ce que Thomas écrit sapientia ${ }^{10}$, sapientiel. Et pour établir ce caractère, de manière remarquable, Aristote et ses catégories disparaissent presque: une fois la fonction du sage aristotélicien énoncée (ordonner et juger), la place se structure par des énoncés pauliniens (1Co 3,10 et Rm 1,19) et augustiniens (De Trinitate 12,14) signalant son enracinement dans la Parole de Dieu communiquée et révélée. Performance théologique structurée, délimitée, localisée et temporalisée par une hyper-performance divine ${ }^{11}$ ! Excès et inachèvements sont à l'ordre du jour, incomplétudes de tous ordres, contamination des ordres... Et si les protocoles structurant la profession théologienne sur le modèle d'un métier et d'un travail, sur le modèle de la production d'un discours et d'ouvrages à partager — sans oublier l'effet en retour sur l'engagement, le temps du travail étudiant - si ces protocoles avaient pour but de jouer la situation paradoxale à la fois d'énoncer de manière maîtrisée ce qui ne se laisse pas maîtriser, ce qu'il y a d'excédant, ce qu'il pourrait y avoir de frustrant dans cette situation, et d'en énoncer le caractère déstabilisant pour l'institution nécessaire à cette énonciation?

\section{Conclusion}

Si et lorsque l'université devient le lieu d'une communauté œuvrante, estce que, en son sein et/ou en ses marges, les pratiquants de théologie ne

9. Summa theologice, Ia, q. 1, a. 4.

10. Summa theologice, Ia, q. 1, a. 6.

11. Summa theologice, Ia, q. 1, a. 6, ad 3. 
formeraient pas, virtuellement du moins, une communauté "désœuvrée » au sens de Jean-Luc Nancy (2004) ? Pour être de/à l'université, la théologie doit-elle, peut-elle, de manière constitutive, prendre le rythme de la production de l'œuvre, de l'article? Si cela est réglé par l'autorité universitaire, unifiante pour tous les départements ou, encore, par le gouvernement et autres bailleurs de fonds, qu'en est-il alors, encore, de l'existence de l'université et de la possibilité de l'organisation du lieu théologique? Cela repose autrement la question médiévale du lieu en fonction du rapport docteurs/étudiants/questions sur fond de désir et de désir réfléchi, énoncé comme tel, d'une préférence professée pour du raisonnable. Quels horizons attirent désormais? Qui et sur quel espace d'expérience? Quelles attentes chez les professeurs et les étudiants? Comment se croisent-elles lorsque et si elles se croisent? Et à quel(s) prix puisque, en bien des sens, de la vérité, cela a un/du prix (Hénaff 2002)? Qui le paye?

Il ne s'agit certes pas de penser recréer à partir de rien des «espaces théologiques ", de faire table rase des facultés ou départements qui existent. Le «modèle " présenté ici à partir de Thomas d'Aquin est un instrument heuristique pour forcer à faire ressortir la double logique qui structure déjà toujours tout lieu: celle de la rationalité calculatrice avec la valence de postulation qu'elle contient (demande, désir) et celle, «inconditionnelle de l'inconditionné ", pour reprendre les catégories derridiennes une dernière fois (2003, 195-200). Il ne s'agit pas de choisir l'une ou l'autre, l'une contre l'autre, mais de choisir de nous en tenir à vivre et à œuvrer dans le désœuvrement que permet le croisement délibéré de ces deux perspectives. Impossible ? La constitution de "lieux" théologiques relèverait de l'hyperéthique et de ses apories, des apories des choix, des décisions sur de l'indécidable... La constitution du lieu théologique, en tant qu'exprimant un rapport exigeant entre des professeurs confessants et des étudiant(e)s désirant professer, devient alors un "événement ». Événement où se jouerait le devenir des rationalités, le devenir désiré et imprévisible des acteurs s'engageant dans le geste qui permettrait cet événement même. Événement qui permettrait un avenir pour ces lieux.

\section{Références}

Bourdieu, P. (1989a), Homo Academicus, Paris, Minuit (Sens commun). (1989b), La noblesse d'État: grandes écoles et esprit de corps, Paris, Minuit (Sens commun). 
Bourdieu, P. et J.-C. Passeron (1970), La reproduction: Éléments d'une théorie du système d'enseignement, Paris, Minuit (Sens commun).

Boyle, L.E. (1982), The Setting of the Summa theologix of Saint Thomas, Toronto, Pontifical Institute of Medieval Studies (The Etienne Gilson Series 5).

Collège de Philosophie (1979), Philosophie de l'université. L'idéalisme allemand et la question de l'université, textes de Schelling, Fichte, Schleiermacher, Humboldt, Hegel / trad. par G. Goffin, J.-F. Courtine, L. Ferry, A. Laks, O. Masson, A. Renaut et J. Rivelaygue, Paris, Payot.

De Libera, A. (2003), Raison et foi: Archéologie d'une crise d'Albert le Grand à Jean-Paul II, Paris, Seuil (L'ordre philosophique).

Derrida, J. (1990), Du droit à la philosophie, Paris, Galilée (La philosophie en effet).

- (2001), L’Université sans condition, Paris, Galilée (Incises).

- (2003), Voyous, Paris, Galilée (La philosophie en effet).

Derrida, J. et G. Vattimo (1996), La religion, Paris, Seuil.

Fenves, P., dir. (1998), Raising the Tone of Philosophy. Late Essays by Immanuel Kant. Transformative Critique by Jacques Derrida, Baltimore, John Hopkins University Press.

Haskins, C.H. (1990), The Rise of Universities, Ithaca, Cornell University Press.

Hénaff, M. (2002), Le prix de la vérité: le don, l'argent, la philosophie, Paris, Seuil (La couleur des idées).

Idinopulos, T. et B. Wilson, dir. (1998), What Is Religion? Origins, Definitions, and Explanations, Leiden / Boston / Cologne, Brill (Studies in the History of Religions. Numen Book Series), vol. 81.

Idinopulos, T. et E.A. Yonna, dir. (1994), Religion and Reductionism. Essays on Eliade, Segal, and the Challenge of the Social Sciences for the Study of Religion, Leiden / New York / Cologne, Brill (Studies in the History of religions. Numen Book Series), vol. 62.

Le Goff, J. (1977), Pour un autre Moyen Âge: temps, travail et culture, Paris, Gallimard (Bibliothèque d'histoire). (1999), Un autre Moyen Âge, Paris, Gallimard (Quarto).

Lyotard, J.-F. (1984), Le différend, Paris, Minuit (Critique). 
McCool, G.A. (1989), Ninetheenth Century Scholsaticism: The Search for a Unitary Method, New York, Fordham University Press.

(1992), From Unity to Pluralism: The Internal Evolution of Thomism, New York, Fordham University Press.

McCutcheon, R.T. (1995), "The Category «Religion» in Recent Publications: A Critical Survey», Numen, 42, p. 284-309.

(1997), Manufacturing Religion. The Discourse on Sui Generis Religion and the Politics of Nostalgia, Oxford / New York, Oxford University Press.

(2001), Critics, Not Caretakers. Redescribing the Public Study of Religion, Albany, State University of New York Press (Issues in the Study of Religion).

Mulcharey, M. (1996), "The Dominican Studium System and the University of Europe in the Thirteenth Century ", dans J. Hamesse, dir., Manuels, programmes de cours et techniques d'Enseignement dans l'université médiévale, Louvain-la-Neuve, Publications de l'Institut d'Études Médiévales, p. 277-325.

Nancy, J.-L. (2004), La communauté désouvrée, Paris, Christian Bourgois (Détroits).

Rashdall, H. (1936), The Universities of Europe in the Middle Ages, vol. 1 : Salerno, Bologna and Paris, London, Clarendon Press.

Verger, J. (1997), L'essor de l'université au XIII e siècle, Paris, Cerf (Initiations au Moyen-Âge).

\section{Résumé}

Afin de construire un protocole heuristique pour questionner les manières de parler des «lieux de la théologie» en rapport avec l'université, cet article propose un parcours inspiré de Derrida. Ayant installé le questionnement épistémologique autour des verbes "professer » et "confesser ", il fait remarquer la complexité des rapports entre ces fonctions. Il inscrit ensuite ces rapports sur le double horizon historique des réflexions allemandes sur l'université à l'orée du XIX ${ }^{\mathrm{e}}$ s. et de l'université médiévale et ses marges. Ce double horizon déplace le questionnement vers les relations entre les professeurs et les étudiants, relations portées par le désir et la promesse de raison comme constitutifs de lieux théologiques dépassant les cadres institués de la faculté de théologie pour se retrouver en jeu dans toute l'université. 


\section{Abstract}

In order to propose a heuristic protocol to understand critically the notion of "theological space» in the University, this article proposes an argument inspired by Derrida. The argument is made around the verbs "profess " and "confess" and their complex relations. These are then reinterpreted on the horizon of the discussions concerning the University in early XIX ${ }^{\text {th }}$ century Germany and, also, of the ones surrounding medieval universities and their margins. This reinterpretation changes the focus: the relations between professors and students come to the fore with the desires and promises of rationality constituting them and constitutive of the theological character of any space of learning at work in University, beyond the limits of any faculty of theology. 